\title{
Panchromatic study of the first galaxies with large ALMA programs
}

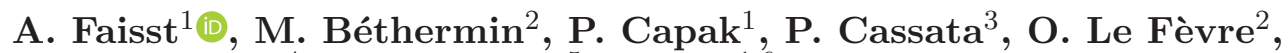 \\ D. Schaerer ${ }^{4}, J$. Silverman ${ }^{5}$, L. Yan $^{1,6}$ and the $A L P I N E$ team \\ ${ }^{1}$ IPAC, California Institute of Technology, 1200 East California Boulevard, \\ Pasadena, CA 91125, USA \\ email: afaisst@ipac.caltech.edu \\ ${ }^{2}$ Laboratoire d'Astrophysique de Marseille UMR 7326, 13388, Marseille, France \\ ${ }^{3}$ University of Padova, Department of Physics and Astronomy Vicolo Osservatorio 3, \\ 35122, Padova, Italy \\ ${ }^{4}$ Observatoire de Genève, Université de Genève $51 \mathrm{Ch}$. des Maillettes, \\ 1290 Versoix, Switzerland \\ ${ }^{5}$ Kavli Institute for the Physics and Mathematics of the Universe, \\ The University of Tokyo Kashiwa, Chiba 277-8583, Japan \\ ${ }^{6}$ Caltech Optical Observatories, Cahill Center for Astronomy and Astrophysics \\ 1200 East California Boulevard, Pasadena, CA 91125, USA
}

\begin{abstract}
Thanks to deep optical to near-IR imaging and spectroscopy, significant progress is made in characterizing the rest-frame UV to optical properties of galaxies in the early universe $(z>4)$. Surveys with Hubble, Spitzer, and ground-based facilities (Keck, Subaru, and VLT) provide spectroscopic and photometric redshifts, measurements of the spatial structure, stellar masses, and optical emission lines for large samples of galaxies. Recently, the Atacama Large (Sub) Millimeter Array (ALMA) has become a major player in pushing studies of high redshift galaxies to far-infrared wavelengths, hence making panchromatic surveys over many orders of frequencies possible. While past studies focused mostly on bright sub-millimeter galaxies, the sensitivity of ALMA now enables surveys like ALPINE, which focuses on measuring the gas and dust properties of a large sample of normal main-sequence galaxies at $z>4$. Combining observations across different wavelengths into a single, panchromatic picture of galaxy formation and evolution is currently and in the future an important focus of the astronomical community.
\end{abstract}

Keywords. Galaxy: formation, Galaxy: evolution, galaxies: ISM, dust, extinction, ISM: evolution, ISM: kinematics and dynamics, ISM: evolution, infrared: galaxies, surveys

\section{Overview}

Galaxies evolve significantly over cosmic time in both internal as well as external properties. The cosmic star formation rate (SFR) density of the early universe increases rapidly to a redshift $z=2$ (Figure 1) and galaxies in the early universe double their stellar mass at $>10$ times faster rates compared to today (Faisst et al. 2016a). This steep mass growth goes in hand with a significant increase of the dust and metal content (Maiolino et al. 2008; Bouwens et al. 2009; Faisst et al. 2016b). In addition, star-forming galaxies evolve from lumpy and turbulent (dominated by gas inflow, mergers, and star-forming clumps) to smooth disks as cosmic time progresses (Förster Schreiber et al. 2011).

In only 500 Myrs between redshifts $4<z<6$, galaxies significantly increase their stellar, dust, and gas mass as well as metal content and in addition change their structure by building up the first stellar disks. Hence, this early growth phase is an important link 



Figure 1. Left: SFR density across cosmic time (see Madau \& Dickinson (2014)). ALPINE targets galaxies in the early growth phase, a transition phase between primordial and mature galaxy formation where galaxies substantially increase their stellar mass, dust, and metal content. Right: Fraction of flux density emitted by galaxies directly in the UV (top shading), by UV-selected galaxies in the far-IR (middle shading), and by galaxies only probed in blind far-IR surveys (bottom shading). Data from Bouwens et al. (2009).

between primordial (during the Epoch of Reionization at $z>4$ ) and mature (at the peak of cosmic SFR density at $z \sim 2-4$ ) galaxy formation.

To date, more than 1000 galaxies have been identified at $4<z<6$ by photometric redshifts, color-color selections, and narrow-band observations targeting Ly $\alpha$ (see review by Dayal et al. 2017). Several hundred galaxies have been confirmed spectroscopically. Large surveys with HST specifically focus on the galaxies' UV properties including their luminosity function, UV dust obscuration, and morphology (e.g., Bouwens et al. 2015; Shibuya et al. 2016). Observations with Spitzer at $3-5 \mu \mathrm{m}$ (rest-frame optical) are used to determine robust stellar masses and to estimate SFRs and stellar mass growth rates from optical emission lines such as $\mathrm{H} \alpha$ (Davidzon et al. 2017; Faisst et al. 2016a). In addition, deep rest-frame UV spectroscopy from 8-10 meter telescopes (e.g., Keck and VLT) characterize UV emission lines (such as Ly $\alpha$ or He II) and provide important constraints on metallicity from the measurement of various absorption features (Ando et al. 2007; Faisst et al. 2016b).

These large UV to optical surveys lay the foundation for a comprehensive panchromatic study over many orders of frequencies with the newest and next-generation facilities. The ALPINE survey with ALMA is a current example of this. The combination of ALMA's sensitivity and efficiency with carefully pre-selected samples from complete optical surveys opens doors for the first large-sample study of dust and gas properties of galaxies in the early growth phase at $z>4$.

\section{ALPINE: A Textbook Example of a Panchromatic Study}

The ALMA Large Program to Investigate [C II] at Early Times is a 70-hour ALMA cycle 5 program (\#2017.1.00428.L) to carry out targeted Band-7 observations of 122 main-sequence galaxies in the early growth phase at $4<z<6$ (Figure 1). ALPINE is a diverse, multi-national collaboration of $\sim 45$ scientists, led by the PI-team of O. Le Fèvre (PI), A. Faisst (North America science lead), M. Béthermin (ALMA data reduction lead), P. Cassata, P. Capak, D. Schaerer, J. Silverman, and L. Yan. As of today 


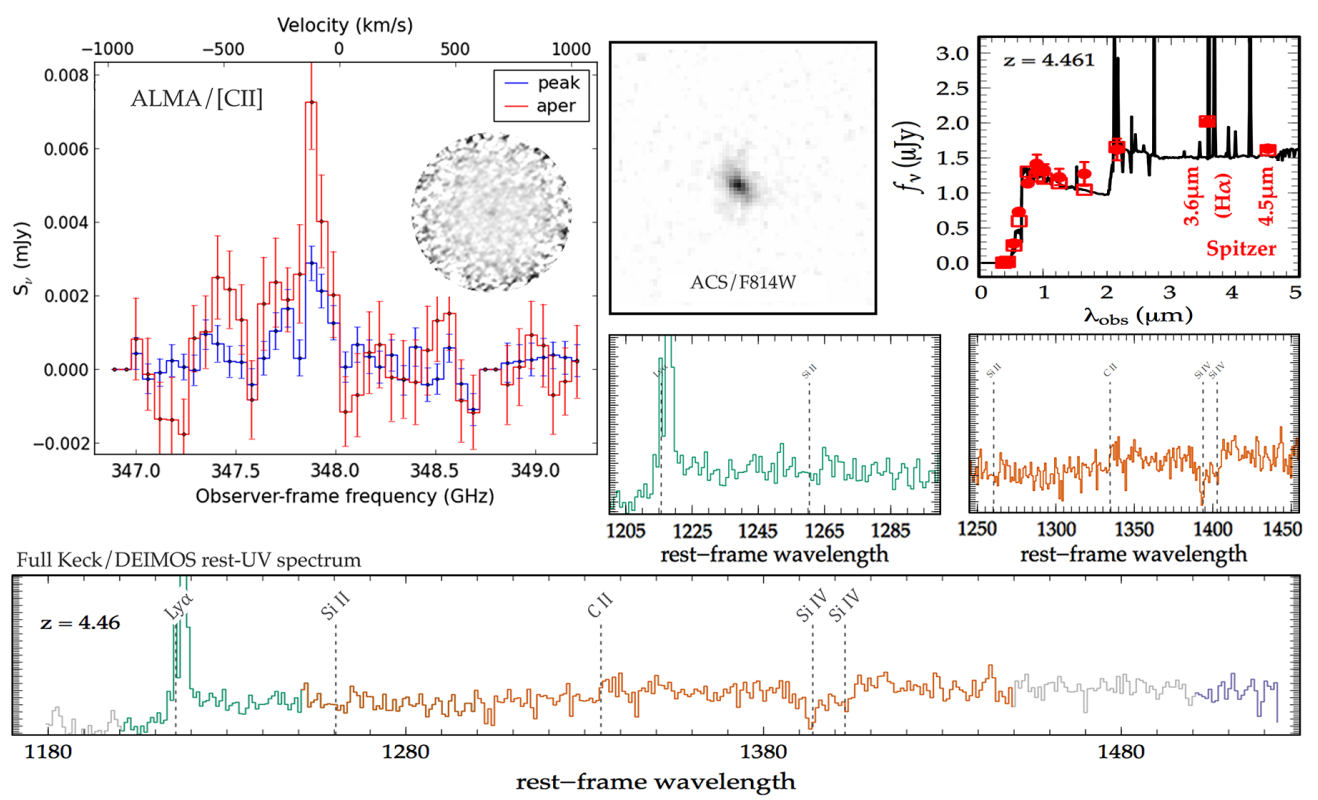

Figure 2. Demonstration of the power of panchromatic data on a galaxy from ALPINE. Clockwise: [C II] emission and dust continuum from ALMA; HST imaging; multi-band SED and $\mathrm{H} \alpha$ from Spitzer color; rest-frame UV spectrum from Keck/DEIMOS (Ly $\alpha$ and absorption lines).

(December 2018), 85\% of the data has been acquired and reduced. For more information on $A L P I N E$, we refer to future survey papers.

ALPINE maximizes its efficiency and impact by building upon a carefully pre-selected sample in the Cosmic Evolution Survey (COSMOS, Scoville et al. 2007) and the Extended Chandra Deep Field South (E-CDFS, Giacconi et al. 2002) fields, well studied fields at UV to near-IR wavelengths. The sample is drawn from large spectroscopic surveys undertaken at Keck (Hasinger et al. 2018) and VLT (Le Fèvre et al. 2015), including galaxies with diverse spectroscopic properties in rest-frame UV emission and absorption lines. This approach leads to a complete probe of a diverse galaxy population above $\mathrm{SFR} \sim 10 \mathrm{M}_{\odot} / \mathrm{yr}$ in a stellar mass range of $9<\log \left(M / \mathrm{M}_{\odot}\right)<11$. By following up UV-selected galaxies, ALPINE probes more than $80 \%$ of the total missing flux density at these redshifts, while blind surveys with ALMA cover the remaining less than 20\% (Figure 1). In combination with its multi-wavelength ancillary data, ALPINE is a textbook example of one of the first large panchromatic surveys probing galaxies in the early Universe (Figure 2).

\subsection{ALPINE Science in a Nutshell}

ALPINE builds upon its successful small-scale precursor study based on 10 galaxies at $z \sim 5.5$ (Riechers et al. 2014; Capak et al. 2015) and reaches a similar sensitivities (continuum: $50 \mu \mathrm{Jy}$ or $\log \left(L_{\mathrm{FIR}} / \mathrm{L}_{\odot}\right) \sim 10.3$; line: $\mathrm{S}_{\mathrm{CII}} \Delta v \sim 0.05 \mathrm{Jy} \mathrm{km} / \mathrm{s}$ or $\left.\log \left(L_{\mathrm{CII}} / \mathrm{L}_{\odot}\right) \sim 7.5\right)$ and resolution $\left(0.5^{\prime \prime}-0.7^{\prime \prime}\right)$. Its main goal is to quantify the evolution of obscured star-formation, the gas and dust content, and gas kinematics of galaxies in the early universe via $\left[\mathrm{C}_{\mathrm{II}}\right]$ emission and far-IR continuum measurements at rest-frame $\sim 150 \mu \mathrm{m}$. $\left[\mathrm{C}_{\mathrm{II}}\right]$ is one of the dominant coolants, hence one of the strongest far-IR lines. In addition the atmospheric transmission is high, making these observations favorable. ALPINE combines several science goals and is designed to answer the following questions. 
How does the total star-formation evolve at $4<z<6$ ? ALPINE provides a comprehensive study of the total SFR density at these redshifts from UV and far-IR continuum measurements. Independent SFRs from far-IR, [C II], and $\mathrm{H} \alpha$ (derived from Spitzer colors, see Faisst et al. 2016a) provide the first measurement of the SFR versus stellar mass main-sequence on different timescales of star-formation at these redshifts.

Is $\left[\mathrm{C}_{\mathrm{II}}\right]$ a reliable SFR indicator at high- $z$ ? The prevalence of [C $\left.\mathrm{II}\right]$ emission in highredshift galaxies is a promising tool to estimate SFRs of far-IR continuum faint galaxies. ALPINE allows to calibrate this locally well established (De Looze et al. 2014) relation at $z>4$ where galaxies are more metal poor.

How dusty are galaxies in the early universe? Small-sample studies of the IRX $-\beta$ relation, which relates the total dust extinction to the line-of-sight dust obscuration, suggest diverse dust properties at $z \sim 5$ (Faisst et al. 2017). ALPINE's large sample constrains the diversity and studies its causes by a comprehensive analysis of its ancillary data.

How does the gas fraction evolve at $z>4$ ? ALPINE constrains gas masses from farIR continuum, [C II], and dynamical mass measurements and measures gas kinematics from $\left[\mathrm{C}_{\mathrm{II}}\right]$ profiles. It is the first study to trace the evolution of gas mass out to $z=6$.

ALPINE unites current UV to far-IR facilities and will in the future build a benchmark sample for various simulations and set the basis for follow-up observations with the James Webb Space Telescope and other next-generation ground- and space-based facilities.

\section{Conclusions}

Large photometric and spectroscopic surveys at UV to near-IR wavelengths provide a solid basis to build a full panchromatic sample over the next couple of years. Importantly, these samples build on a variety of galaxies, which makes optimally planned targeted follow-up observations with ALMA and later JWST and TMT/E-ELT efficient in covering a significant amount of the parameter space of galaxy properties. A current example is ALPINE, which is specifically built to extend the wavelength coverage of a carefully chosen large spectroscopic sample with ground- and space-based multi-wavelength photometric ancillary data. This makes it to one of the currently largest panchromatic samples to study the formation and evolution of normal main-sequence galaxies in the early Universe. In the future, the ALPINE sample will provide a solid basis for follow-up observations with various next-generation facilities.

\section{References}

Ando, M., Ohta, K., Iwata, I., Akiyama, M., Aoki, K., Tamura, N., et al. 2007, PASJ, 59, 717

Bouwens, R. J., Illingworth, G. D., Franx, M., Chary, R.-R., Meurer, G. R., Conselice, C. J., Ford, H., Giavalisco, M., et al. 2009, ApJ, 705, 936

Bouwens, R. J., Illingworth, G. D., Oesch, P. A., Trenti, M., Labbé, I., Bradley, L., Carollo, M., van Dokkum, P. G., et al. 2015, ApJ, 803, 34

Capak, P. L., Carilli, C., Jones, G., Casey, C. M., Riechers, D., Sheth, K., Carollo, C. M., Ilbert, O., et al. 2015, Nature, 522, 455

Dayal, P. \& Ferrara, A. 2017, Physics Reports, 780, 1

Davidzon, I., Ilbert, O., Laigle, C., Coupon, J., McCracken, H. J., Delvecchio, I., Masters, D., Capak, P., et al. 2017, A\&A, 605, A70

De Looze, I., Cormier, D., Lebouteiller, V., Madden, S., Baes, M., Bendo, G. J., Boquien, M., Boselli, A., et al. 2014, A\& A, 568, A62

Faisst, A. L., Capak, P., Hsieh, B. C., Laigle, C., Salvato, M., Tasca, L., Cassata, P., Davidzon, I., et al. 2016, ApJ, 821, 122

Faisst, A. L., Capak, P. L., Davidzon, I., Salvato, M., Laigle, C., Ilbert, O., Onodera, M., Hasinger, G., et al. 2016, ApJ, 822, 29

Faisst, A. L., Capak, P. L., Yan, L., Pavesi, R., Riechers, D. A., Barišić, I., Cooke, K., Kartaltepe, J. S., et al. 2017, ApJ, 847, 21 
Förster Schreiber, N. M., Shapley, A. E., Erb, D. K., Genzel, R., Steidel, C. C., Bouché, N., Cresci, G., Davies, R., et al. 2011, ApJ, 731, 65

Giacconi, R., Zirm, A., Wang, J., Rosati, P., Nonino, M., Tozzi, P., Gilli, R., Mainieri, V., et al. 2002, ApJS, 139, 369

Hasinger, G., Capak, P., Salvato, M., Barger, A. J., Cowie, L. L., Faisst, A., Hemmati, S., Kakazu, Y., et al. 2018, ApJ, 858, 77

Le Fèvre, O., Tasca, L. A. M., Cassata, P., Garilli, B., Le Brun, V., Maccagni, D., Pentericci, L., Thomas, R., et al. 2015, A\&BA, 576, A79

Madau, P. \& Dickinson, M. 2014, ARAA, 52, 415

Maiolino, R., Nagao, T., Grazian, A., Cocchia, F., Marconi, A., Mannucci, F., Cimatti, A., et al. 2008, A\&A A 488, 463

Riechers, D. A., Carilli, C. L., Capak, P. L., Scoville, N. Z., Smolčić, V., Schinnerer, E., Yun, M., Cox, P., et al. 2014, ApJ, 796, 84

Scoville, N., Aussel, H., Brusa, M., Capak, P., Carollo, C. M., Elvis, M., Giavalisco, M., Guzzo, L., et al. 2007, ApJS, 172, 1

Shibuya, T., Ouchi, M., Kubo, M. \& Harikane, Y. 2016, ApJ, 821, 72

\section{Discussion}

T. Gото: Is there a correlation between IRX $-\beta$ and geometry of the galaxies?

A. FAIsst: We generally see that galaxies with a high $L_{\mathrm{FIR}} / L_{\mathrm{UV}}$ are more compact. Galaxies with a low ratio look more clumpy and irregular. However, the sample size is not yet big enough to draw any conclusions, but we will with ALPINE.

D. Burgarella: Why would you expect galaxies to move from the local galaxy region (i.e. Meurer) to below the SMC one in the IRX $-\beta$ diagram?

A. FAISST: We expect lower dust masses at high- $z$, so galaxies will likely evolve downwards. For $\beta$, that question is still open, spatial geometry effects might play a role.

F. Fontanot: I have a question about your initial sample of 10 galaxies. Do you have a mass estimate for these objects? And if so, do you see a significant difference in mass or redshift range between the dust-rich and the dust-poor population?

A. FAIsst: The current sample (10 galaxies) is too small to see trends on the IRX- $\beta$ diagram. Also, the mass-range is pretty small. The masses are around $\log \left(M / \mathrm{M}_{\odot}\right)=$ $10 \pm 0.3$. Also, the redshift range in the current sample is small $(5.2<z<5.6)$. We currently do not see any significant trends of dust attenuation with mass and redshift. However, this is something we will study with ALPINE. Specifically, it covers more than a magnitude in stellar mass $\left(9.5<\log \left(M / \mathrm{M}_{\odot}\right)<11.5\right)$ and a large redshift range $(4.4<z<5.8)$. If there are trends with stellar mass and redshift, we will likely see it in ALPINE.

D. Rosario: Does the data allow a comparison of $\mathrm{H} \alpha$ and $[\mathrm{C}$ II]?

A. FAIsst: $\mathrm{H} \alpha$ can be estimated from the excess in the Spitzer/IRAC bands at 3.6 and $4.5 \mu \mathrm{m}$. A statistical approach is possible. A direct comparison can be made in individual cases. This is something the team wants to do, for studying gas physics and comparison of different star-formation tracers. 